\title{
Propriedades físicas de Latossolo Vermelho após milho e plantas de cobertura cultivadas em monocultivo ou consorciada
}

\author{
Physical properties of Latossolo Vermelho after maize and cover crop cultivated in monoculture or \\ consortium
}

Propiedades físicas del Latossolo Vermelho después de maíz y plantas de cubierta cultivadas en monocultura o consorcio

Recebido: 23/06/2021 | Revisado: 29/06/2021 | Aceito: 21/07/2021 | Publicado: 28/07/2021

\author{
Henrique Marsaro Thomé \\ ORCID: https://orcid.org/0000-0002-3564-0214 \\ Universidade Estadual do Oeste do Paraná, Brasil \\ E-mail: hmarsarothome@gmail.com \\ Edleusa Pereira Seidel \\ ORCID: https://orcid.org/0000-0001-8821-473X \\ Universidade Estadual do Oeste do Paraná, Brasil \\ E-mail: seideledleusa8@gmail.com \\ Jandrei Matheus Stein \\ ORCID: https://orcid.org/0000-0003-4307-8636 \\ Universidade Estadual do Oeste do Paraná, Brasil \\ E-mail: jandreistein@hotmail.com \\ Renan Pan \\ ORCID: https://orcid.org/0000-0003-0243-7305 \\ Universidade Estadual do Oeste do Paraná, Brasil \\ E-mail: renanpan45@hotmail.com \\ Luane Laíse Oliveira Ribeiro \\ ORCID: https://orcid.org/0000-0002-2913-7620 \\ Universidade Estadual do oeste do Paraná, Brasil \\ E-mail: luanelaiseifpa@ hotmail.com
}

\begin{abstract}
Resumo
O presente trabalho teve por objetivo avaliar as propriedades físicas de um LATOSSOLO VERMELHO Eutroférrico após o cultivo de milho segunda safra e plantas de cobertura cultivadas em monocultivo ou consorciadas. O trabalho foi desenvolvido na área da cooperativa Copagril em Marechal Cândido Rondon-PR. O delineamento experimental foi em blocos casualizados constituído de seis tratamentos: (T1) Milho monocultivo (controle), (T2) Milho em consórcio com braquiária (Brachiaria ruzizienses), (T3) Braquiária em monocultivo, (T4) Crotalária (Crotalaria spectabilis) em monocultivo, (T5) Milho em consórcio com Crotalaria, (T6) Aveia preta (Avena sativum) em monocultivo. Até 0,10 $\mathrm{m}$ o efeito dos cultivos de milho, Brachiaria ruziziensis, Crotalária spectabilis e aveia preta foram semelhantes para a densidade e porosidade do solo. Enquanto, na profundidade de 0,10 a $0,20 \mathrm{~m}$ a Brachiaria ruziziensis foi mais eficiente em aumentar a macroporosidade em relação a Crotalaria spectabilis. A crotalária cultivada em monocultivo não foi capaz de reduzir a resistência do solo à penetração.
\end{abstract}

Palavras-chave: Densidade do solo; Plantas de cobertura; Porosidade do solo; Qualidade física.

\begin{abstract}
The present research aimed to evaluate the physical properties of an LATOSSOLO VERMELHO Eutroférrico after the cultivation of maize second crop and cover crops grown in monoculture or intercropping. This research was developed in the area of the Copagril cooperative in Marechal Cândido Rondon-PR. The experimental design was in randomized blocks consisting of six treatments: (T1) Maize monoculture (control), (T2) Maize intercropped with Brachiaria (Brachiaria ruzizienses), (T3) Brachiaria in monoculture, (T4) Crotalaria (Crotalaria spectabilis) in monoculture, (T5) Corn intercropped with Crotalaria, (T6) Black oat (Avena sativum) in monoculture. Up to $0.10 \mathrm{~m}$ the effect of corn, Brachiaria ruziziensis, Crotalária spectabilis and black oat crops were similar for soil density and porosity. While, at depth from 0.10 to $0.20 \mathrm{~m}$, Brachiaria ruziziensis was more efficient in increasing macroporosity than Crotalaria spectabilis. The Crotalaria spectabilis cultivated in monoculture was not able to reduce soil resistance to penetration.
\end{abstract}

Keywords: Soil density; Cover crops; Soil porosity; Physical quality. 


\begin{abstract}
Resumen
El presente trabajo tuvo como objetivo evaluar las propiedades físicas de un LATOSSOLO VERMELHO Eutroférrico después del cultivo de maíz de segunda cosecha y cultivos de cobertura en monocultivo o intercalado. El trabajo se desarrolló en el área de la cooperativa Copagril en Marechal Cândido Rondon-PR. El diseño experimental fue en bloques al azar que consistió en seis tratamientos: (T1) Monocultivo de maíz (control), (T2) Maíz intercalado con Brachiaria (Brachiaria ruzizienses), (T3) Brachiaria en monocultivo, (T4) Crotalaria (Crotalaria spectabilis) en monocultivo, (T5) Maíz intercalado con Crotalaria, (T6) Avena negra (Avena sativum) en monocultivo. Hasta $0.10 \mathrm{~m}$ el efecto de los cultivos de maíz, Brachiaria ruziziensis, Crotalária spectabilis y Avena negra fue similar en densidad y porosidad del suelo. Mientras que, a una profundidad de 0,10 a $0,20 \mathrm{~m}$, Brachiaria ruziziensis fue más eficiente para aumentar la macroporosidad que Crotalaria spectabilis, el cáñamo Crotalaria cultivado en monocultivo no pudo reducir la resistencia del suelo a la penetración.
\end{abstract}

Palabras clave: Densidad del suelo; Cultivos de cobertura; Porosidad del suelo; Calidad física.

\title{
1. Introdução
}

Muitos problemas de degradação dos recursos naturais são influenciados pela ação antrópica devido ao manejo inadequado do solo, tornando este, suscetível à ação de processos erosivos. As intensas e constantes operações com equipamentos pesados podem afetar a estrutura do solo. Também induz a uma rápida decomposição e destruição da matéria orgânica (Troian, et al., 2020) e consequentemente leva a compactação do solo com redução do crescimento radicular e permeabilidade (Jeloudar Taleshian, et al., 2018). Isso resultará em diminuição no rendimento das culturas (Marques, 2019).

Neste sentido, a adoção de sistemas de manejo que visem à sustentabilidade, tornam-se cada dia mais essenciais para que se mantenha a qualidade e sustentabilidade dos sistemas agrícolas, melhorando a estrutura física do ambiente edáfico, aumentando a infiltração de água no solo e aeração, sustentação do crescimento vegetal; e, consequentemente melhorando a produtividade agrícola e a qualidade do solo (Sanchez, et al., 2014).

Nessa perspectiva, o uso de plantas de cobertura torna-se um dos fundamentos para a manutenção da qualidade e sustentabilidade dos sistemas produtivos (Santos, 2012). Segundo Severiano et al. (2010), as plantas de cobertura são uma ótima alternativa para descompactar e melhorar a estrutura do solo, propiciando a formação de bioporos, aumentando o movimento de água e a difusão de gases no solo.

É importante salientar que o produtor não necessariamente deve abrir mão de sua fonte de renda para cultivar plantas de cobertura, podendo este utilizar do sistema de consórcio entre plantas de cobertura e espécies comerciais. A cultura do milho é uma das plantas mais favoráveis para ser utilizada nesse sistema de cultivo devido a sua grande adaptação fisiológica (Seidel, et al., 2017). No consórcio, a produção de massa seca aportada ao solo geralmente é maior do no monocultivo, o que irá favorecer e promover ao longo dos anos, a manutenção das propriedades do solo e aumento na produtividade das culturas (Mottin, 2019). O cultivo de milho consorciado com plantas de cobertura traz vários benefícios para o agricultor. Ao mesmo tempo as plantas de coberturas melhoram as propriedades físicas do solo e ainda obtém-se uma renda com a venda dos grãos de milho (Conceição, et al., 2005).

As propriedades físicas do solo são afetadas pelas diferentes práticas de manejo, e algumas são utilizadas como indicadoras de qualidade física do solo (QFS), destacando-se entre elas: a resistência a penetração (RP), densidade (Ds), porosidade do solo e a estabilidade de agregados do solo (Pacheco \& Cantalice, 2011), as quais sofrem influência direta ou indireta do teor de MO presente no solo (Demattê, et al., 2011). A resistência do solo a penetração (RP), densidade do solo (Ds) e porosidade estão relacionadas ao bom desenvolvimento e estabelecimento das culturas (Roboredo, et al., 2010). A qualidade física assume importância na avaliação do grau de degradação do solo e na identificação de práticas de uso sustentáveis, isso porque a mesma afeta a parte química e biológica do solo (Santos, et al., 2011).

Desta forma, a implantação de plantas de cobertura consorciadas com milho safrinha pode ser uma ferramenta viável para a melhoria das propriedades físicas do solo, sendo uma opção de fonte de renda para o produtor do Oeste do Paraná. Neste 
sentido, o trabalho teve como objetivo avaliar as propriedades físicas de um LATOSSOLO VERMELHO Eutroférrico após o cultivo de milho safrinha e plantas de cobertura cultivadas em monocultivo ou consorciadas.

\section{Metodologia}

\subsection{Caracterização da área}

O experimento foi conduzido no campo experimental da Cooperativa Agrícola Mista Rondon (COPAGRIL) situada no município de Marechal Cândido Rondon, Oeste do Paraná, Brasil. O clima da região, de acordo com a classificação de Köppen, é do tipo Cfa, subtropical com verões quentes, geadas pouco frequentes e concentração de chuvas nos meses de verão, sem estação seca definida caracterizando-se como região subtropical (Alvarez, et al., 2013). O solo da área experimental é classificado como LATOSSOLO VERMELHO Eutroférrico (LVef), textura muito argilosa, com relevo suave ondulado (Santos, et al., 2013). A temperatura média da região é inferior a $18{ }^{\circ} \mathrm{C}$, e a precipitação média de $1840 \mathrm{~mm}$.

\subsection{Delineamento, condução e avaliações}

O delineamento experimental utilizado foi o de blocos casualizados composto por seis tratamentos distribuídos em 4 repetições. Os tratamentos foram: (T1) - Milho monocultivo (testemunha), (T2) - Milho em consórcio com braquiária (Brachiaria ruzizienses), (T3) - Braquiária em monocultivo, (T4) - Crotalária (Crotalaria spectabilis) em monocultivo, (T5) Milho em consórcio com Crotalaria, (T6) - Aveia preta (Avena sativum) em monocultivo.

As parcelas apresentavam $2 \mathrm{~m}$ de largura e $20 \mathrm{~m}$ de comprimento, totalizando uma área experimental de $40 \mathrm{~m}^{2}$. A semeadura do milho foi realizada em fevereiro de 2019, com espaçamento de $0,50 \mathrm{~m}$ com uma densidade média de 3 sementes por metro. Aos 21 dias após a emergência do milho nos tratamentos consorciados foram semeadas manualmente na entrelinha a braquiária e a crotalária. A densidade de semeadura das plantas de cobertura foi de 10 e $30 \mathrm{~kg} \mathrm{ha}^{-1}$, respectivamente.

Para os tratamentos com braquiária e crotalária em monocultivo, a semeadura foi realizada em março de 2019, no mesmo dia em que foi semeada no consórcio, com espaçamento entre linhas de $0,25 \mathrm{~m}$. A aveia preta, foi semeada em maio de 2019, com espaçamento entre linhas de 0,25 m e uma densidade de $60 \mathrm{~kg}$ ha-1 de sementes.

Após a colheita do milho foram realizadas coletas de amostras indeformadas de solo para avaliação das propriedades físicas: densidade do solo (Ds) (pelo método da estufa com anéis de peso e volume conhecido), porosidade total (Pt), macro e microporosidade (pelo método da mesa de tensão e estufa), resistência do solo a penetração (Rp) (determinado através do penetrômetro digital).

Foram coletadas em dois pontos de cada parcela amostras indeformadas de solo nas profundidades de $0-0,05 \mathrm{~m} ; 0,05-$ $0,10 \mathrm{~m}$ e $0,10-0,20 \mathrm{~m}$. As amostras foram encaminhadas para laboratório onde foram pesadas, saturadas por 24 horas, em seguida levadas para mesa de tensão por 24 horas, para serem novamente pesadas e obter-se, então, o volume de macroporos.

Na sequência, estas foram levadas para estufa de circulação forçada à $105^{\circ} \mathrm{C}$ até massa constante para obtenção dos dados referentes a microporosidade e umidade volumétrica. Para a determinação da resistência do solo à penetração das raízes (RP) utilizou-se o penetrômetro FALKER® PGL 1020, na profundidade de 0 a $40 \mathrm{~cm}$, com o aparelho regulado para realizar leituras a cada $1 \mathrm{~cm}$, as quais foram posteriormente estratificadas para cada $5 \mathrm{~cm}$, a partir da superfície do solo até a profundidade de $40 \mathrm{~cm}$. Foram realizadas cinco medições por parcela. A umidade do solo de cada área foi determinada e o solo estava na capacidade de campo (CC), sendo a umidade média de $25,77 \%$. 


\subsection{Análise dos dados}

Os dados foram tabulados e submetidos ao teste de normalidade e posteriormente foi feito a análise de variância pelo teste F. Em seguida os dados foram submetidos ao teste de Tukey a 5\% de probabilidade de erro, com auxílio do software SISVAR.

\section{Resultados e Discussão}

A densidade média do solo nas três profundidades analisadas foi de $1,32 \mathrm{Mg} \mathrm{m}^{-3}$ (Tabela 1). Este valor está abaixo do nível considerado crítico para a maioria das culturas de grãos que é de $1,40 \mathrm{Mg} \mathrm{dm}^{-3}$ (Reichert, et al., 2009). Acima deste valor, ocorre condições restritivas ao crescimento e desenvolvimento do sistema radicular; pois, há redução na infiltração e transporte de água no solo e diminuição das trocas gasosas entre o solo e a atmosfera (Fonseca, et al., 2007).

Tabela 1. Valores médios para densidade do solo (Ds), macroporosidade (Ma), microporosidade (Mi) e porosidade total (Pt) nas profundidades de 0,0-0,05; 0,05-0,10; 0,10-0,20 m de um LATOSSOLO VERMELHO Eutroférrico sob diferentes manejos com plantas de cobertura. Marechal Cândido Rondon - PR, 2019.

\begin{tabular}{|c|c|c|c|c|}
\hline \multirow[t]{2}{*}{ Manejo } & $\begin{array}{c}\text { Densidade } \\
\text { (Ds) }\end{array}$ & $\begin{array}{l}\text { Macro } \\
\text { (Ma) }\end{array}$ & $\begin{array}{l}\text { Micro } \\
\text { (Mi) }\end{array}$ & \multirow[t]{2}{*}{ Porosidade $(\mathrm{Pt})$} \\
\hline & $\mathrm{Mg} \mathrm{m}^{-3}$ & \multirow{2}{*}{\multicolumn{2}{|c|}{------------- $\mathrm{m}^{3} \mathrm{~m}^{-3}$----------- }} & \\
\hline & & Profundidade & & \\
\hline & & $0,00-0,05 \mathrm{~m}$ & & \\
\hline Milho monocultivo & $1,33^{\mathrm{ns}}$ & $0,086^{\mathrm{ns}}$ & $0,048^{\mathrm{ns}}$ & $0,056^{\text {ns }}$ \\
\hline Milho+ B. ruziziensis & 1,28 & 0,088 & 0,050 & 0,059 \\
\hline Brachiaria ruziziensis & 1,24 & 0,076 & 0,055 & 0,062 \\
\hline Crotalaria spectabilis & 1,26 & 0,096 & 0,052 & 0,062 \\
\hline Milho+Crotalaria $s$. & 1,27 & 0,011 & 0,047 & 0,057 \\
\hline \multirow[t]{3}{*}{ Aveia Preta } & 1,33 & 0,084 & 0,049 & 0,057 \\
\hline & & Profundidade & & \\
\hline & & $0,05-0,010 \mathrm{~m}$ & & \\
\hline Milho monocultivo & $0,014^{\mathrm{ns}}$ & $0,093^{\text {ns }}$ & $0,049^{\text {ns }}$ & $0,058^{\mathrm{ns}}$ \\
\hline Milho+ B. ruziziensis & 0,014 & 0,063 & 0,055 & 0,061 \\
\hline Brachiaria ruziziensis & 0,013 & 0,011 & 0,050 & 0,061 \\
\hline Crotalaria spectabilis & 0,013 & 0,073 & 0,044 & 0,061 \\
\hline Milho+Crotalaria $s$. & 0,013 & 0,011 & 0,046 & 0,057 \\
\hline \multirow[t]{3}{*}{ Aveia Preta } & 0,013 & 0,010 & 0,047 & 0,058 \\
\hline & & Profundidade & & \\
\hline & & $0,010-0,020 \mathrm{~m}$ & & \\
\hline Milho monocultivo & $1,45^{\mathrm{ns}}$ & $0,064 \mathrm{ab}$ & $0,499^{\text {ns }}$ & $0,544^{\mathrm{ns}}$ \\
\hline Milho+ B. ruziziensis & 1,36 & $0,074 \mathrm{ab}$ & 0,543 & 0,587 \\
\hline Brachiaria ruziziensis & 1,34 & $0,110 \mathrm{a}$ & 0,512 & 0,622 \\
\hline Crotalaria spectabilis & 1,32 & $0,063 \mathrm{~b}$ & 0,522 & 0,585 \\
\hline Milho+Crotalaria $s$. & 1,38 & $0,082 \mathrm{ab}$ & 0,473 & 0,555 \\
\hline Aveia Preta & 1,33 & $0,079 \mathrm{ab}$ & 0,492 & 0,571 \\
\hline
\end{tabular}

ns: Não significativo ao nível de 5\% de probabilidade de erro. Médias seguidas da mesma letra minúscula na coluna não diferem entre si ao nível de 5\% de probabilidade de erro. T1 - Milho monocultivo (testemunha); T2 - Milho em consórcio com Brachiaria ruziziensis; T3 Brachiaria ruziziensis; $\mathrm{T} 4-$

Crotalaria spectabilis; T5 - Milho em consórcio com Crotalaria spectabilis; T6 - Aveia preta.

Fonte: Autores. 
Não houve efeito significativo das plantas de cobertura na densidade do solo, microporosidade e porosidade total nas profundidades avaliadas (Tabela 1). Geralmente, alterações na densidade do solo necessita de mais tempo de cultivos com plantas de cobertura, enquanto a microporosidade é pouco afetada pelo manejo. A microporosidade tem maior correlação com a textura do que com os teores de carbono orgânico do solo, ou seja, com o manejo adotado (Viana et al., 2011). Estes resultados podem ser corroborados pelos trabalhos realizados Borghi e Crusciol (2007), Spera et al. (2008) e Seidel et al. (2015) que não obtiveram mudanças significativas nestas propriedades ao trabalhar com plantas de cobertura.

Outro fator que pode ter contribuído para que não se observasse diferenças significativas nessas propriedades é o fato das avaliações serem realizadas imediatamente após a colheita do milho; portanto a matéria seca das plantas de cobertura ainda não estavam decompostas e seus efeitos ainda não observados. Destaca-se também que as espécies utilizadas no consórcio foram plantas de verão, e como foram cultivadas no verão/outono provavelmente tiveram menor crescimento, tanto da parte aérea como de raízes. Esse menor desenvolvimento pode ter contribuído para não observar alterações na densidade e porosidade do solo.

A porosidade total média foi de $0,59 \mathrm{~m}^{3} \mathrm{~m}^{-3}$ e pode ser considerada adequada para uma boa aeração e infiltração de água (Tabela 1). De acordo com Andrade, Stone \& Silveira (2009) para que o solo apresente boa aeração e retenção de água ele deve apresentar porosidade total próxima ou acima de $0,50 \mathrm{~m}^{3} \mathrm{~m}^{-3}$, sendo que $1 / 3$ deve ser de macroporos e $2 / 3$ de microporos.

As plantas de cobertura foram eficientes em aumentar a macroporosidade (Ma) na profundidade de $0,10-0,20 \mathrm{~m}$. A braquiária cultivada em monocultivo apresentou a maior macroporosidade $\left(0,11 \mathrm{~m}^{3} \mathrm{~m}^{-3}\right)$; enquanto, a crotalária a menor $(0,063$ $\mathrm{m} \mathrm{m}^{-3}$ ). A crotalária apesar de seu sistema radicular ser profundo tipo pivotante o volume de raiz é menor do que o produzido pela Brachiaria ruziziensis que é fasciculado. Apesar das condições climáticas não serem as mais adequadas para o cultivo destas espécies a braquiária conseguiu se desenvolver bem e favoreceu a formação de macroporos em maior profundidade o que influenciará diretamente na capacidade de drenagem deste solo. A macroporosidade do solo na área com as demais plantas de cobertura foi inferior a $0,10 \mathrm{~m}^{3} \mathrm{~m}^{-3}$.

Segundo Mottin (2019), a braquiária tem grande potencial para promover melhorias na macroporosidade do solo devido principalmente a presença de raízes volumosas e bem desenvolvidas. Além disso, suas raízes finas podem ter se decomposto formando bioporos e melhorando a agregação do solo refletindo na macroporosidade. A braquiária pertence a família Poaceae e a literatura destaca sua capacidade em promover melhorias físicas do solo. Ainda Mottin et al. (2018) constatou que as plantas de cobertura do solo da família Poaceae promoveram maior volume de macroporos quando comparado as plantas da família Fabaceae.

Na Figura 1 são apresentados os valores médios para a resistência do solo a penetração (RP). A maior RP até a profundidade de 0,25 m foi na área cultivada com Crotalária spectabilis, o que é coerente; pois, foi a menor densidade de solo (Tabela 1). Neste tratamento a RP ficou acima do valor crítico para a maioria das culturas que é de 2,0 Mpa. Demonstrando que o solo se encontra compactado (Tuzzin de Moraes, et al., 2016).

Camadas compactadas limitam o desenvolvimento radicular em profundidade, e consequentemente o volume de solo explorado para extração de água (Nosalewicz \& Lipiec, 2014) e nutrientes (Schnepf, Leitner \& Klepsch, 2012). Assim, em uma situação onde há uma camada de solo muito compactada.

Enquanto, a menor RP até a profundidade de $0,13 \mathrm{~m}$ foi observada no tratamento com braquiária em monocultivo ou em consórcio com o milho. As raízes da braquiária são fasciculadas e tem bom desenvolvimento o que resulta em maior volume de solo explorado e pode, portanto, romper camadas compactadas trazendo melhorias físicas do solo (Moura, 2012). 
Na profundidade de 0,30 a 0,40 m na área cultivada com aveia preta foi a que obteve a maior RP (2,20 Mpa). Esse resultado é em função do sistema radicular desta cultura que se concentra na profundidade de $0,20 \mathrm{~m}$. Por isso, não se observou o efeito descompactador de suas raízes.

Figura 1. Variação da resistência do solo à penetração (RP) de um LATOSSOLO VERMELHO em função de diferentes modalidades de cultivo de milho, Brachiaria ruziziensis, Crotalária spectabilis, Aveia Preta BRS 139. T1 - Milho monocultivo (testemunha); T2 - Milho em consórcio com Brachiaria ruziziensis; T3 - Brachiaria ruziziensis; T4 - Crotalaria spectabilis; T5 - Milho em consórcio com Crotalaria spectabilis; T6 - Aveia preta.

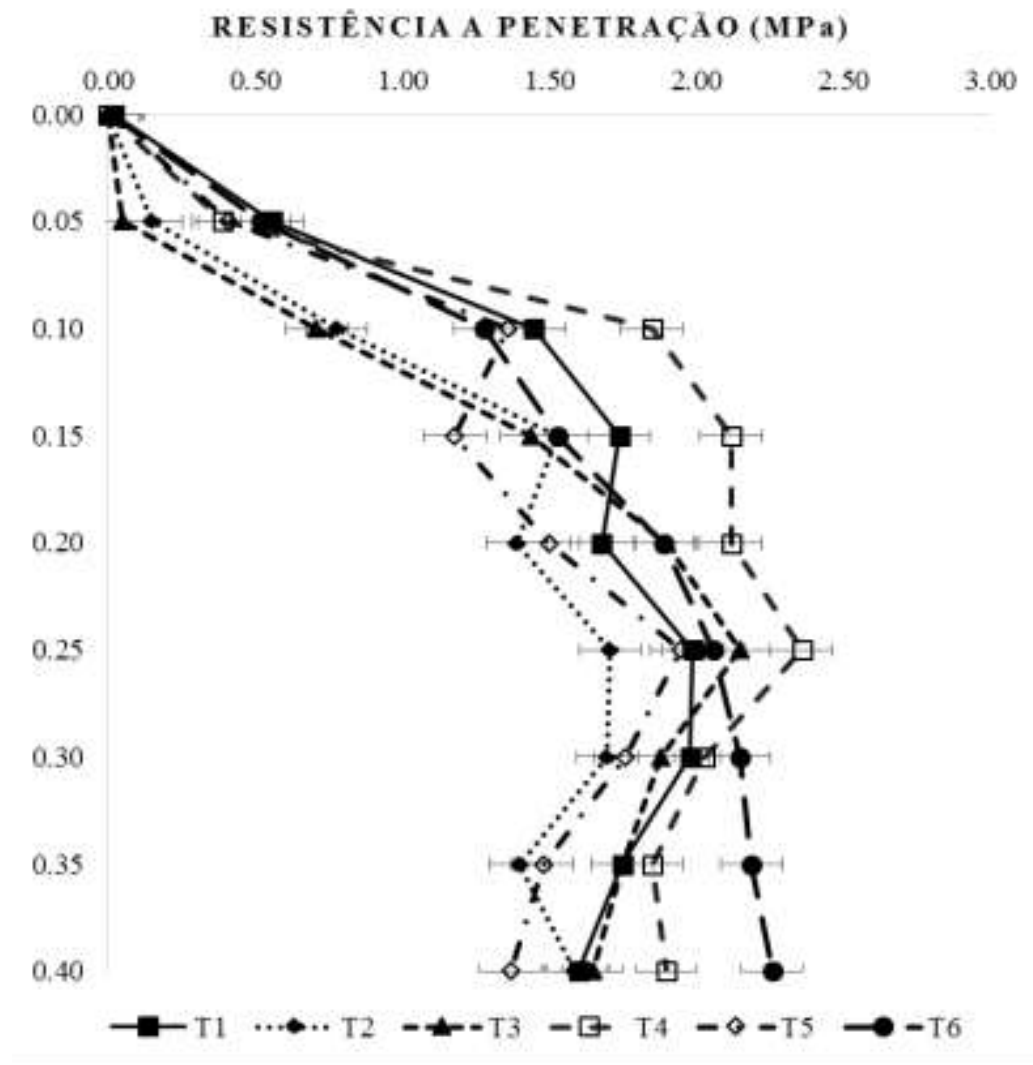

Fonte: Autores.

Para Anschau (2018), a camada de 0,10 a 0,30 m foi a que apresentou valores mais elevados quando avaliou-se da resistência do solo à penetração (3,5 a 4,5 Mpa). Assim como em trabalho realizado por Rosset et al. (2014), que observou valores superiores ao limite critico ( $2 \mathrm{Mpa}$ ), o que sugere a ocorrência de camadas subsuperficiais compactadas, o que pode afetar de forma negativa o desenvolvimento das culturas (Dimassi, et al., 2013).

\section{Conclusão}

Até $0,10 \mathrm{~m}$ o efeito dos cultivos de milho, Brachiaria ruziziensis, Crotalária spectabilis e aveia preta foram semelhantes para a densidade e porosidade do solo. Enquanto, na profundidade de 0,10 a 0,20 m a Brachiaria ruziziensis foi mais eficiente em aumentar a macroporosidade em relação a Crotalaria spectabilis.

A crotalária cultivada em monocultivo não foi capaz de reduzir a resistência do solo à penetração. 


\section{Referências}

Alvarez, C. A., Stape, J. L., Sentelhas, P. C., Gonçalves, J. L. M., \& Sparovek, G. (2013). Köppens's climate classification map for Brazil. Meteorologische Zeitschrift, 22(1), 711-728. 10.1127/0941-2948/2013/0507

Andrade, R. S., Stone, L.F., \& Silveira, P. M. (2009). Culturas de cobertura e qualidade física de um Latossolo em plantio direto. Revista Brasileira de Engenharia Agrícola e Ambiental, 13, 411- 418. https://doi.org/10.1590/S1415-43662009000400007

Anschau, K. A., Seidel, E. P., Mottin, M. C., Lerner, K. L., Francziskowski, M. A., \& Herrmann, D. da R. (2018). Propriedades físicas do solo, características agronômicas e produtividade da soja em sucessão a plantas de cobertura. Scientia Agraria Paranaensis, 17(3), 293-299. http://erevista.unioeste.br/index.php/scientiaagraria/article/view/19702

Borghi, E., \& Crusciol, C. A. C. (2007). Produtividade de milho, espaçamento e modalidade de consorciação com Brachiaria brizantha em sistema plantio direto. Pesquisa Agropecuária Brasileira, 42(2), 163-171. https://doi.org/10.1590/S0100-204X2007000200004

Conceição, P. C., Amado, T. J. C., Mielniczuk, J., \& Spagnollo, E. (2005). Qualidade do solo em sistemas de manejo avaliada pela dinâmica de matéria orgânica e atributos relacionados. Revista Brasileira de Ciência do Solo,29(5), 777-788. https://doi.org/10.1590/S0100-06832005000500013

Demattê, J. A. M., Bortoletto, M. A. M., Vasques, G. M., \& Rizzo, R. (2011). Quantificação de matéria orgânica do solo através de modelos matemáticos utilizando colorimetria no sistema Munsell de cores. Bragantia, 70(3), 590-597. https://doi.org/10.1590/S0006-87052011005000006

Dimassi, B., Cohanb, J. P., Labreuche, J., \& Mary, B. (2013). Changes in soil carbon and nitrogen following tillage conversion in a long-term experiment in Northern France. Agriculture, Ecosystems \& Environment, 169, 12-20. https://doi.org/10.1016/j.agee.2013.01.012

Fonseca, G. C., Carneiro, M. A. C., da Costa, A. R., de Oliveira, G. C.., \& Balbino, L. C. (2007). Atributos físicos, químicos e biológicos de Latossolo Vermelho Distrófico de cerrado sob duas rotações de cultura. Revista Agropecuária Tropical, 37, 22-30. https://www.redalyc.org/articulo.oa?id=253020279005

Jeloudar Taleshian, F., Ghajar Sepanlou, M., \& Emadi, M. (2018). Impact of land use change on soil erodibility. Global Journal of Environmental Science and Management, 4(1), 59-70. 10.22034/GJESM.2018.04.01.006

Marques, J. F. (2019). Custos da erosão do solo em razão dos seus efeitos internos e externos à área de produção agrícola. Revista de Economia e Sociologia Rural, 36(1), 61-80. https://www.revistasober.org/journal/resr/article/5da2a1e00e882523472371d3

Mottin, M. C. Caracterização química da matéria orgânica, propriedades físicas do solo e produtividade de milho de segunda safra consorciado com plantas de cobertura e soja em sucessão. (2019). Tese de Doutorado em Agronomia.

Mottin, M. C., Seidel, E. P., Fey, E., Vanelli, J., Alves, A. L., Richart, A., Frandoloso, J. F., Anschau, K. A., \& Francziskowski, M. A. (2018). Biomass Productivity and Physical Properties of the Soil after Cultivation of Cover Plant in the Autumn and Winter. American Journal of Plant Sciences, 09, 775-788. https://doi.org/10.4236/ajps.2018.94061

Moura, J. B., Marasca, I., Meneses, L. A. da S., Pires, W. M., \& Medeiros, L. C. (2012). Resistência a penetração do solo em pastagem cultivada com Brachiaria decumbens sob aplicação de dejetos líquidos suínos e cama de frango. Global Science and Technology, 5(3). https://www.researchgate.net/publication/281271191_Resistencia_a_penetracao_do_solo_em_pastagem_cultivada_com_Brachiaria_decumbens_sob_aplicaca o_de_dejetos_liquidos_suinos_e_cama_de_frango

Nosalewicz, A., \& Lipiec, J. (2014). The effect of compacted soil layers on vertical root distribution and water uptake by wheat. Plant and Soil, 375(2), 229240. 10.1007/s11104-013-1961-0

Pacheco, E. P., \& Cantalice, J. R. B. (2011). Análise de trilha no estudo dos efeitos de atributos físicos e matéria orgânica sobre a compressibilidade e resistência à penetração de um Argissolo cultivado com cana-de-açúcar. Revista Brasileira de Ciência do Solo, 35(2), 403-415. https://doi.org/10.1590/S010006832011000200011

Reichert, J. M., Suzuki, L. E. A. S., Reinert, D. J., Horn, R., \& Håkansson, I. (2009). Reference bulk density and critical degree-of-compactness for no-till crop production in subtropical highly weathered soils. Soil and Tillage Research, 102(2), 242-254. https://doi.org/10.1016/j.still.2008.07.002

Roboredo, D., Maia, J. C. S., Oliveira, O. J., \& Roque, C. G. (2010). Uso de dois penetrômetros na avaliação da resistência mecânica de um Latossolo Vermelho Distrófico. (2010). Revista $\quad$ Engenharia 307-314. https://www.scielo.br/j/eagri/a/rLfHTRsXdySDGqWfyrhVmgv/?format=pdf\&lang=pt

Rosset, J. S., Lana, M. do C., Pereira, M. G., Schiavo, J. A., Rampim, L., Sarto, M. V. M., \& Seidel, E. P. (2014). Carbon stock, chemical and physical properties of soils under management systems with different deployment times in western region of Paraná, Brazil. Semina, 35(6), 3053-3072. 10.5433/1679$0359.2014 \mathrm{v} 35 \mathrm{n} 6 \mathrm{p} 3053$

Schnepf, A., Leitner, D., \& Klepsch, S. (2012). Modeling Phosphorus Uptake by a Growing and Exuding Root System. Vadose Zone Journal, 11(3), 1-10. https://doi.org/10.2136/vzj2012.0001

Sanchez, E., Maggi, M. F., Genú, A. M., \& Müller, M. M. L. (2014). Propriedades físicas do solo e produtividade de soja em sucessão a plantas de cobertura de inverno. Magistra, 26, 48. https://magistraonline.ufrb.edu.br/index.php/magistra/article/view/462

Santos, G. G., Silveira, P. M., Marchão, R. L., Petter, F. A., \& Becquer, T. (2012). Atributos químicos e estabilidade de agregados sob diferentes culturas de cobertura em Latossolo do cerrado. Revista Brasileira de Engenharia Agrícola e Ambiental, 16(11), 1171-1178. https://doi.org/10.1590/S141543662012001100005

Santos, G. G., Silva, E. M., Marchão, R. L., Silveira, P. M., Bruand, A., James, F., \& Becquer, T. (2011). Analysis of physical quality of soil using the water retention curve: Validity of the S-index. C. R. Geoscience, 343, 295-301. https://doi.org/10.1016/j.crte.2011.02.001 
Research, Society and Development, v. 10, n. 9, e39210917493, 2021

(CC BY 4.0) | ISSN 2525-3409 | DOI: http://dx.doi.org/10.33448/rsd-v10i9.17493

Santos, H. G., Almeida, J. A., Lumbreras, J. F., Anjos, L. H. C., Coelho, M. R., Jacomine, P. K. T., Cunha, T. J. F., \& Oliveira, V. A. Sistema Brasileiro de Classificação de Solos. $3^{\mathrm{a}}$ ed. Brasília: Empresa Brasileira de Pesquisa Agropecuária.

Seidel, E. P., dos Reis, W., Mottin, M. C., Fey, E., Schneider, A. P. R., \& Sustakowski, M. C. (2017). Evaluation of aggregate distribution and selected soil physical prperties under maize-jack bean intercropping and gypsum rates. African Journal of Agricultural Research, 12(14), 1209-1216. https://academicjournals.org/journal/AJAR/article-full-text-pdf/D98BB4363621

Seidel, E. P., Mattia, V., Mattei, E., \& Corbari, F. (2015). Produção de matéria seca e propriedades físicas do solo na consorciação milho e braquiária. Scientia Agraria Paranaensis, 14(1), 18-24. http://e-revista.unioeste.br/index.php/scientiaagraria/article/view/8226

Severiano, E. C., Oliveira, G. C., Dias Junior, M. S., Costa, K. A. P., Castro, M. B., \& Magalhães, E. N. (2010). Potencial de descompactação de um Argissolo promovido pelo capim-tifton 85. Revista Brasileira Engenharia Agrícola e Ambiental, 14, 39-45. https://www.scielo.br/j/rbeaa/a/pLZwvy35yntHFwB8FB5HBPs/?lang=pt\&format=pdf

Spera, S. T., Denardin, J. E., Escosteguy, P. A. V., Santos, H. P. D., \& Figueroa, E. A. (2008). Dispersão de argila em microagregados de solo incubado com calcário. Revista Brasileira de Ciência do Solo, 32, 2613-2620. https://doi.org/10.1590/S0100-06832008000700002

Troian, D., Rosset, J. S., Martins, L. F. B. N., Ozório, J. M.B., Castilho, S. C. de P., Marra, L. M. (2020). Carbono orgânico e estoque de carbono do solo em diferentes sistemas de manejo. Revista em Agronegócios e Meio Ambiente. 13(4), 1447-1469. https://doi.org/10.17765/2176-9168.2020v13n4p1447-1469

Tuzzin de Moraes, M., Debiasi, H., Carlesso, R., Franchini, J. C., da Silva, V. R., \& da Luz, F. B. (2016). Soil physical quality on tillage and cropping systems after two decades in the subtropical region of Brazil. Soil and Tillage Research, 155, 351-362. http://dx.doi.org/10.1016/j.still.2015.07.015.

Viana, E. T., Batista, M. A., Tormena, C. A., Costa, A. C. S., \& Inoue, T. T. (2011). Atributos físicos e carbono orgânico em Latossolo Vermelho sob diferentes sistemas de uso e manejo. Revista Brasileira de Ciência do Solo, 35(6), 2105-2114. 10.1590/S0100-06832011000600025 